\title{
Acute Mesenterie Venous Thrombosis Induced by Smoking
}

Yigit Mehmet Ozgun', Hale Demir ${ }^{2}$, Gonca Ozgun ${ }^{3}$, Necdet Deniz Tihan ${ }^{4}$, Orhan Bat ${ }^{1}$

From the Department of General Surgery, Istanbul Kanuni Sultan Suleyman Teaching and Research Hospital'; Department of Pathology, Istanbul University Medical Faculty ${ }^{2}$; Department of Pathology, Istanbul Medipol University Medical Faculty ${ }^{3}$; Department of General Surgery, Bursa Sevket Yılmaz Teaching and Research Hospital ${ }^{4}$, Turkey.

\section{Abstract:}

Mesenteric venous thrombosis is the least common cause of acute mesenteric ischemia which is generally difficult to diagnose and can be fatal. Mesenteric venous thrombosis has a variety of causes. Largescale follow-up studies have suggested an association between smoking and the venous thrombosis. In this case report we present a young male who presented to emergency department with acute peritonitis after cigarette over-consumption. He had significant leukocytosis and thrombocytopenia. An emergency laparotomy was performed. Ischemic segment of the ileum with thrombotic mesenteric veins was resected and the patient was discharged uneventfully on the $7^{\text {th }}$ postoperative day.

Key words: Smoking, Peritonitis, Thrombosis, Mesenteric Ischemia, Vascular Diseases, Venous Thrombosis.

\section{Introduction}

Mesenteric venous thrombosis (MVT) accounts for $5-15 \%$ of all acute mesenteric ischemia (AMI) cases. Mortality rates range between $20-50 \%$ depending on time of diagnosis (early vs. late), chronicity of the disease (acute vs. chronic), degree of venous involvement, presence of intestinal ischemia, and the degree of ischemia [1-3]. An underlying primary hypercoagulable state should be sought for especially in young cases with MVT [4]. Some previous studies have shown that smoking independently increases the risk of venous thrombosis [5-7]. We present here a young male patient with bowel ischemia due to acute MVT who had no additional primary hypercoagulable state or pathological blood tests. We suggest that intensive smoking may have caused MVT in this patient.

\section{Case Report}

A 32-year-old male patient admitted to emergency department with complaint of abdominal pain for about 7 days. He had no past medical and surgical history. He was smoking 20 cigarettes per day for 12 years and in the last 10 days he consumed 40 50 cigarettes per day. At his physical examination his vital signs were stable; however, laboratory examination revealed significant leukocytosis

Corresponding Author: Dr. Yigit Mehmet Ozgun

Email: dryigitozgun@gmail.com

Received: January 17,2014 | Accepted: August 19,2014 | Published Online: September 10, 2014 This is an Open Access article distributed under the terms of the Creative Commons Attribution License (creativecommons.org/licenses/by/3.0)

Conflict of interest: None declared | Source of funding: Nil | DOl: http://dx.doi.org/10.17659/01.2014.0086 
and thrombocytopenia (WBC: $234 \times 10^{6} / \mathrm{dL}$, hemoglobin $13.6 \mathrm{~g} / \mathrm{dL}$, platelet $79 \times 10^{6} / \mathrm{dL}$, INR of 1.2). Results of blood biochemistry tests were normal.

Abdominal X-ray revealed valvula conniventes and air-fluid level [Fig.1]. The ultrasound examination demonstrated free fluid in the abdomen. Any further investigations including computed tomography were not performed in view of marked signs of acute peritonitis. An emergency exploration was performed. A serosanguineous peritoneal fluid of $1,000 \mathrm{~mL}$ was observed without signs of perforation. Full-thickness necrosis of an $80 \mathrm{~cm}$ part of the ileum with thrombotic mesenteric veins was identified [Fig.2]. About $100 \mathrm{~cm}$ of ileum including the necrotic segment was resected, followed by end-to-end anastomosis. The pulsation of superior mesenteric artery and its branches was normal. Patient was discharged with no complications on the $7^{\text {th }}$ postoperative day. The pathologic examination revealed edema and hemorrhage at all layers of intestinal wall as well as dilatation and congestion of mesenteric vessels and hemorrhage in lymph nodes. Tests (Factor $V$ Leiden mutation, antithrombin III deficiency, protein $S$ and protein $C$ deficiency, hyperhomocysteinemia, antiphospholipid antibody syndrome, and prothrombin mutations) for thrombotic disorders performed after the surgery, were negative.

\section{Discussion}

The etiologic factors of MVT include hypercoagulopathy (factor-5 Leiden mutation, protein- $\mathrm{C}, \mathrm{S}$ and antithrombin-3 deficiencies, hyperhomocysteinemia, anti-phospholipid antibody syndrome etc), portal vein hypertension, trauma, intra-abdominal sepsis, cirrhosis, pancreatitis, postsplenectomy, malignancy, pregnancy, and use of oral contraceptives. Only $10 \%$ of cases are undiagnosed and classified as idiopathic $[2,4,8]$.

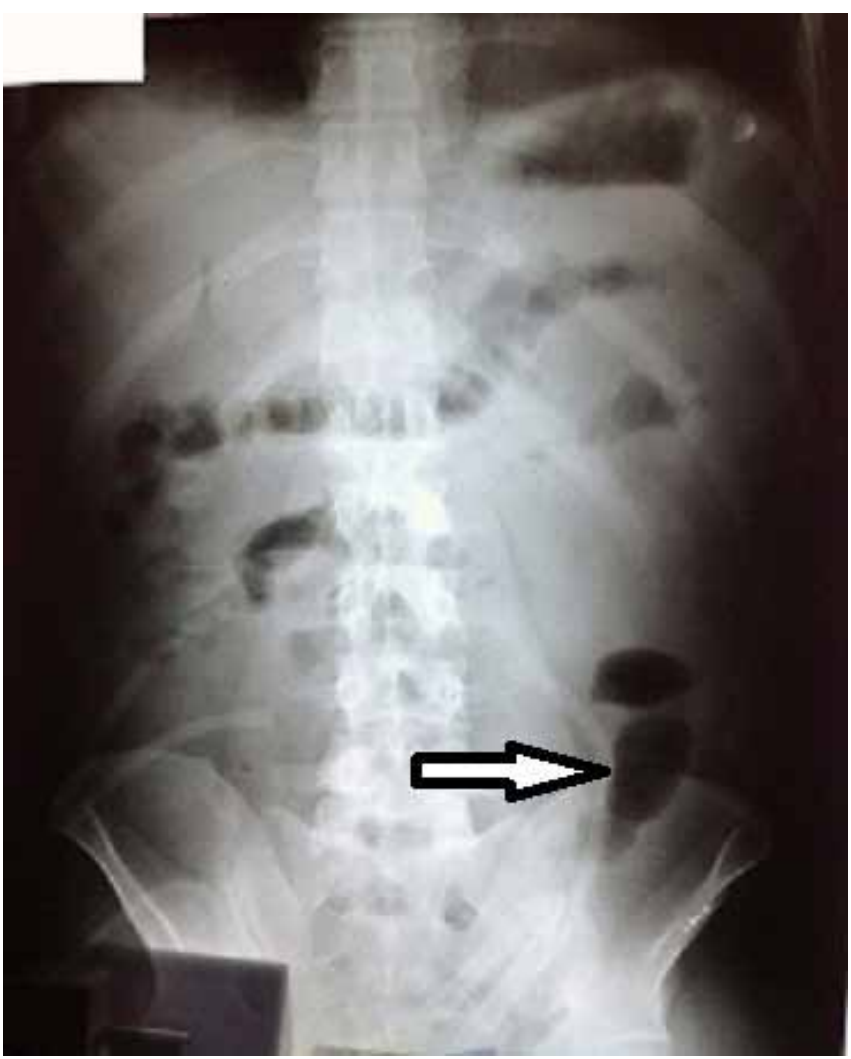

Fig.1: Plain X-ray revealed valvula conniventes and dilated gas filled small intestine loops at the left inferior part of the abdomen.

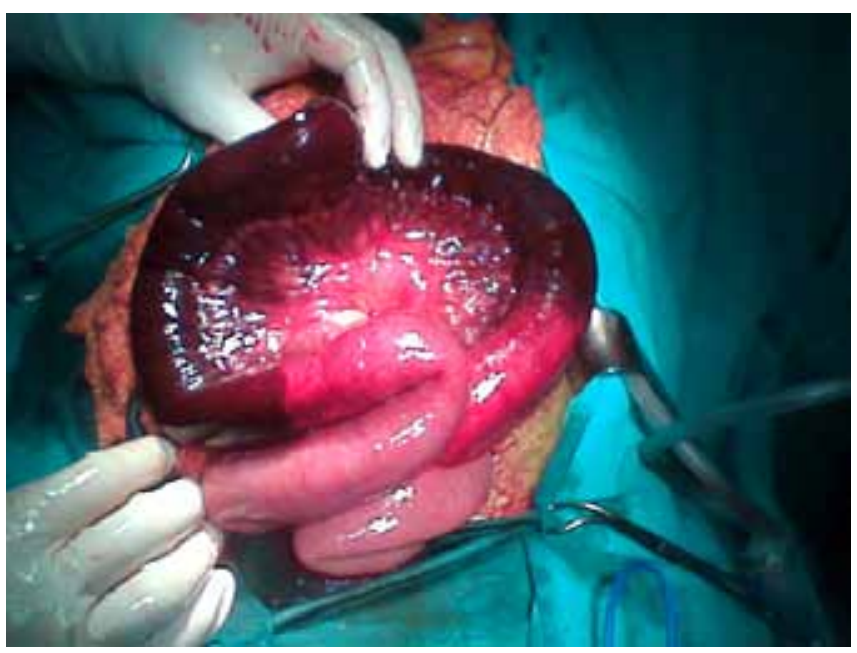

Fig.2: lleum with necrosis. Edema and hemorrhage were seen at the intestinal wall and its mesentery. 
Clinical symptoms of MVT are often nonspecific and cause delays in diagnosis. The most common symptoms are fever, diffuse abdominal pain, bloody stools, and anorexia. The MVT should be kept in mind for early diagnosis, which will reduce the morbidity and mortality and require promptly CT scan $[2,4,9]$. In general, the diagnosis is usually made by obtaining a history of a prodromal period of days to weeks, which is characterized by abdominal pain that is usually out of proportion to physical findings $[2,3]$. Computed tomography (CT) is $90-100 \%$ sensitive for the diagnosis of MVT and guides treatment $[3,10]$.

Computed tomography is more sensitive in diagnosis of venous thrombus than other types of AMI [2]. In acute arterial occlusive ischemia, the bowel wall becomes thinner due to reduced blood flow to appear as the so-called paper-thin-wall. In addition, neither mural edema nor hemorrhage occurs at the bowel wall. In MVT, on the other hand, the bowel wall becomes thicker because of edema and hemorrhage $[2,10]$. The microscopic features of MVT include hyperemia, hemorrhage, and edema of the intestinal wall. In addition, hemorrahagic infarctions may occur due to occlusion of intramural vessels $[2,11]$. In our case, the patient suffered from peritonitis and underwent an emergency laparotomy without preoperative CT scan. In preoperative blood tests he had thrombocytopenia which we considered to be secondary to platelet consumption at the mesenteric veins. A limited number of studies have shown that there is an association between thrombocytopenia and pulmonary embolism. These studies have related thrombocytopenia to platelet consumption and pointed out that the degree of thrombocytopenia indicates the severity of pulmonary embolism [12]. As in our case, preoperative thrombocytopenia can be an indicator for coagulopathic disorders like mesenteric venous thrombosis as well as pulmonary embolism.
Smoking is an independent risk factor for venous thrombosis. Furthermore, it also makes a synergistic effect in the presence of an underlying hypercoagulopathy $[5,6]$. Severinsen et al. in a large prospective study, reported a positive association between smoking and venous thromboembolism in both men and women. They also advocated that smoking exerts an acute effect because former smokers have the same risk as those who never smoked [7]. As our patient had a history of excessive cigarette consumption within a short period of time before the incident, we considered that MVT may have developed due to acute effect of smoking.

Yanar et al. reported a 34-case series of MVT disease. All patients were initially treated with low molecular weight heparin. Patients with peritoneal signs were evaluated with diagnostic laparoscopy and bowel resection was performed when there was an evidence of necrosis in laparotomy. Patients without peritoneal findings during follow-up were treated with warfarin [13]. The treatment of MVT depends on presence of intestinal ischemia. In the absence of intestinal ischemia the treatment includes anticoagulant therapy and observation. If there are signs of peritonitis, a laparoscopic evaluation should be performed to prevent unnecessary laparotomy. In the presence of ischemia or CT findings that do not justify non-operative treatment, a laparotomy and bowel resection is mandatory. If a hypercoagulable state is detected oral anticoagulation therapy (warfarin $5 \mathrm{mg}$ daily) should be started $[2,13]$.

We could not detect any hypercoagulable state in our case, and therefore the anticoagulant therapy was interrupted. As our patient had a history of excessive cigarette consumption within a short period of time before the incident, we considered that MVT may have developed due to acute effect of smoking. Our patient quit smoking and has been under follow-up for over 2 years with no further events or recurrences. 


\section{Conclusion}

To conclude, in cases with acute abdominal pain, MVT should be remembered when there is a history of smoking, especially in presence of underlying hypercoagulopathy and low platelet levels. Further investigations are needed to draw a conclusion that smoking alone may cause MVT.

\section{References}

1. Kalaitzis J, Basioukas P, Karzi E, Markakis C, Liarmakopoulos E, Hadjimarkou A, Rizos S. Smallbowel necrosis complicating a cytomegalovirusinduced superior mesenteric vein thrombosis in an immunocompetent patient: a case report. J Med Case Rep 2012;6:118.

2. Oldenburg WA, Lau LL, Rodenberg TJ, Edmonds $\mathrm{HJ}$, Burger CD. Acute mesenteric ischemia: a clinical review. Arch Intern Med 2004; 164(10):1054-1062.

3. Gaspary MJ, Auten J, Durkovich D, Gable P. Superior mesenteric vein thrombosis mimicking acute appendicitis. West J Emerg Med 2011 ;1 2(2):262-265.

4. Joh JH, Kim DI. Mesenteric and portal vein thrombosis: treated with early initiation of anticoagulation. Eur J Vasc Endovasc Surg 2005;29(2):204-208.

5. Pomp ER, Rosendaal FR, Doggen CJ. Smoking increases the risk of venous thrombosis and acts synergistically with oral contraceptive use. Am J Hematol 2008;83(2):97-102.

6. Tapson VF. The role of smoking in coagulation and thromboembolism in chronic obstructive pulmonary disease. Proc Am Thorac Soc
2005;2(1):71-77.

7. Severinsen MT, Kristensen SR, Johnsen SP, Dethlefsen C, Tjønneland A, Overvad K. Smoking and venous thromboembolism: a Danish followup study. J Thromb Haemost 2009;7(8):1297303.

8. Chang RW, Chang JB, Longo WE. Update in management of mesenteric ischemia. World J Gastroenterol 2006; 1 2(20):3243-3247.

9. Klar E, Rahmanian PB, Bücker A, Hauenstein K, Jauch KW, Luther $B$. Acute mesenteric ischemia: a vascular emergency. Disch Arztebl Int 2012 ; 109(14):249-256.

10. Furukawa A, Kanasaki S, Kono N, Wakamiya $M$, Tanaka T, Takahashi M, Murata K. CT diagnosis of acute mesenteric ischemia from various causes. Am J Roentgenol 2009;192(2):408416.

11. Lin H, Lin CC, Huang WT. Idiopathic superior mesenteric vein thrombosis resulting in small bowel ischemia in a pregnant woman. Case Rep Obstet Gynecol 2011;2011:687250.

12. Ju-Feng Hsiao, Chi-Ming Chu, Chang-Min Chung, Shih-Tai Chang, Chi-Tai Kuo, Jen-Te Hsu. Prognostic Significance of Thrombocytopenia in Acute Pulmonary Embolism. Journal of Internal Medicine of Taiwan 2008;19(6):499-507.

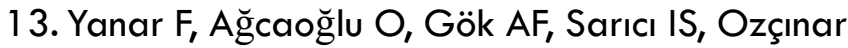
$B$, Aksakal N, Aksoy M, Ozkurt E, Kurtoğlu M. The management of mesenteric vein thrombosis: a single institution's experience. Turkish Journal of Trauma \& Emergency Surgery 2013;19(3):223228. 